\title{
„Meine fotografische Arbeit ist Spiegelbild meines politischen Engagements."
}

\author{
Interview mit der Fotografin Marily Stroux
}

Wie bist Du zum Fotografieren gekommen und was ist Dir bei Deiner Arbeit wichtig?

Ich bin mit Fotografie aufgewachsen. Schon als Kind habe ich zusammen mit meinem Vater fotografiert. Wir hatten sogar eine Dunkelkammer zu Hause. Nach der Geburt meiner Tochter fing ich an, Fotografie beruflich zu betreiben und soziale sowie politische Themen in den Blick zu nehmen. Dabei hat mich die Frage angetrieben, in was für einer Welt mein Kind eigentlich groß wird. Die Fotografie zeigte mir, dass vieles geändert werden muss und ich einen Teil dazu beitragen wollte und konnte. Zum Beispiel als in der Hafenstraße in Hamburg bei einer Räumung tatsächlich Türen aus dem Fenster flogen und keine Öffentlichkeit vor Ort war. Ich habe dann auf Fotos festgehalten, wie die Bewohner*innen zusehen mussten, wie ihre ganzen Sachen einfach aus dem Fenster geworfen, zu Müll erklärt und beschlagnahmt wurden. Wir haben das Foto sofort für ein Flugblatt genutzt, um die Räumung und den Umgang mit den Bewohner*innen öffentlich zu machen. Schon am nächsten Tag wussten viel mehr Menschen, welche Ungerechtigkeit sich hier ereignet hatte. Da wurde mir klar, was Fotografie bewirken kann. Dieses Ereignis motivierte mich, Ungerechtigkeiten auch weiterhin mittels der Fotografie zu dokumentieren.

Kannst Du uns etwas über die Entstehung der Fotoserie „Kinderspiele in der Stadt 1987-2021" erzählen? Was führte zu den Arbeiten und was ist Dir dabei besonders in Erinnerung geblieben? Siehst Du Veränderungen, was das Aufwachsen von Kindern in der Stadt betrifft?

Ich war viel in den Hamburger Vierteln unterwegs, insbesondere in St. Pauli. Meine Kamera hatte ich immer dabei, denn die besten Momente kommen meist überraschend. Du musst also immer mit offenen Augen durch die Stadt laufen. So nahm ich vieles um mich herum sehr bewusst wahr. Situationen, die andere gar nicht bemerken würden, machte ich durch Fotos erst sichtbar. 


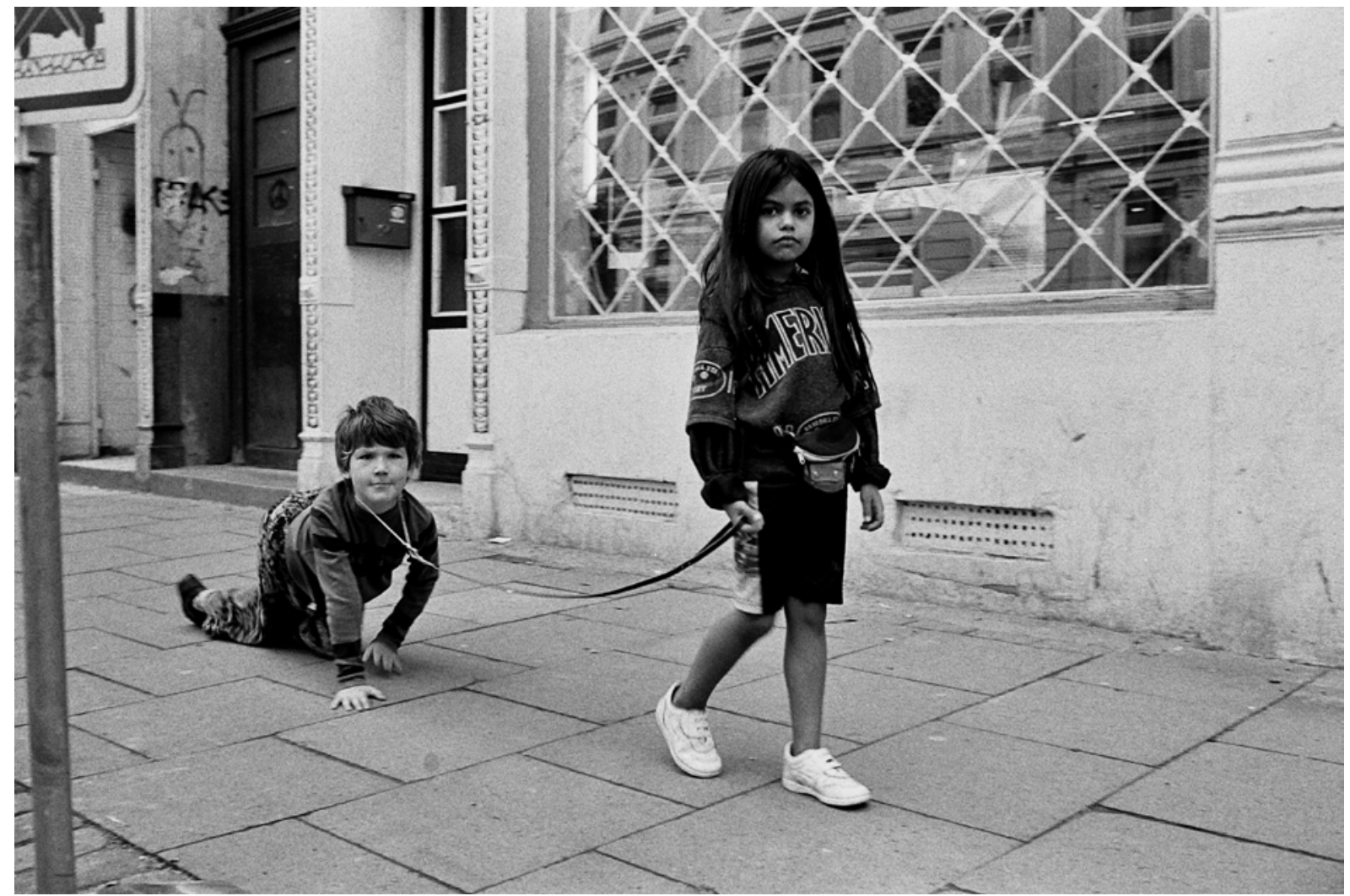

Ein Beispiel: Ich saß in einem Café in der Wohlwillstraße. Während ich aus dem Fenster auf die Straße schaute, ging ein Junge an mir vorbei, der sich von einem Mädchen an der Leine führen ließ. Ich rannte aus dem Café und habe sofort ein Foto gemacht. Die Kinder guckten überrascht und auch etwas streng. Sie spielten in verteilten Rollen Hund und Hundehalterin. Heute, viele Jahre später, beobachte ich, wie die Nachbarkinder in unserem Garten Pferd spielen: Ein Kind hat die Leine um und eines zieht und befielt. Dazwischen liegen 30 Jahre und es sind Kinder aus ganz anderen Verhältnissen - aber alle spielen sie glücklich dieselben Spiele.

Ein weiteres Beispiel ist die Saure-Gurken-Konservendeckel-Schlacht: In der Simon-von-Utrecht-Straße gab es 1994 eine Brache, die als Parkplatz genutzt wurde. Eines Tages kam ich vorbei und sah mehrere Kinder, die euphorisch hunderte Gegenstände in die Luft warfen. Es sah aus wie ein Schlachtfeld, wie eine Kissenschlacht. Ich stieg von meinem Fahrrad und fing an, Fotos zu machen. Ich erkannte, dass die Kinder einen LKW aufgemacht hatten und es Deckel von Konservendosen waren, die da durch die Luft flogen. Als ich das verstanden hatte, kam schon ein schreiender Mann auf uns zugelaufen und wir sind im $\mathrm{Nu}$ alle weggerannt. Ich weiß leider nicht, was danach passierte. 

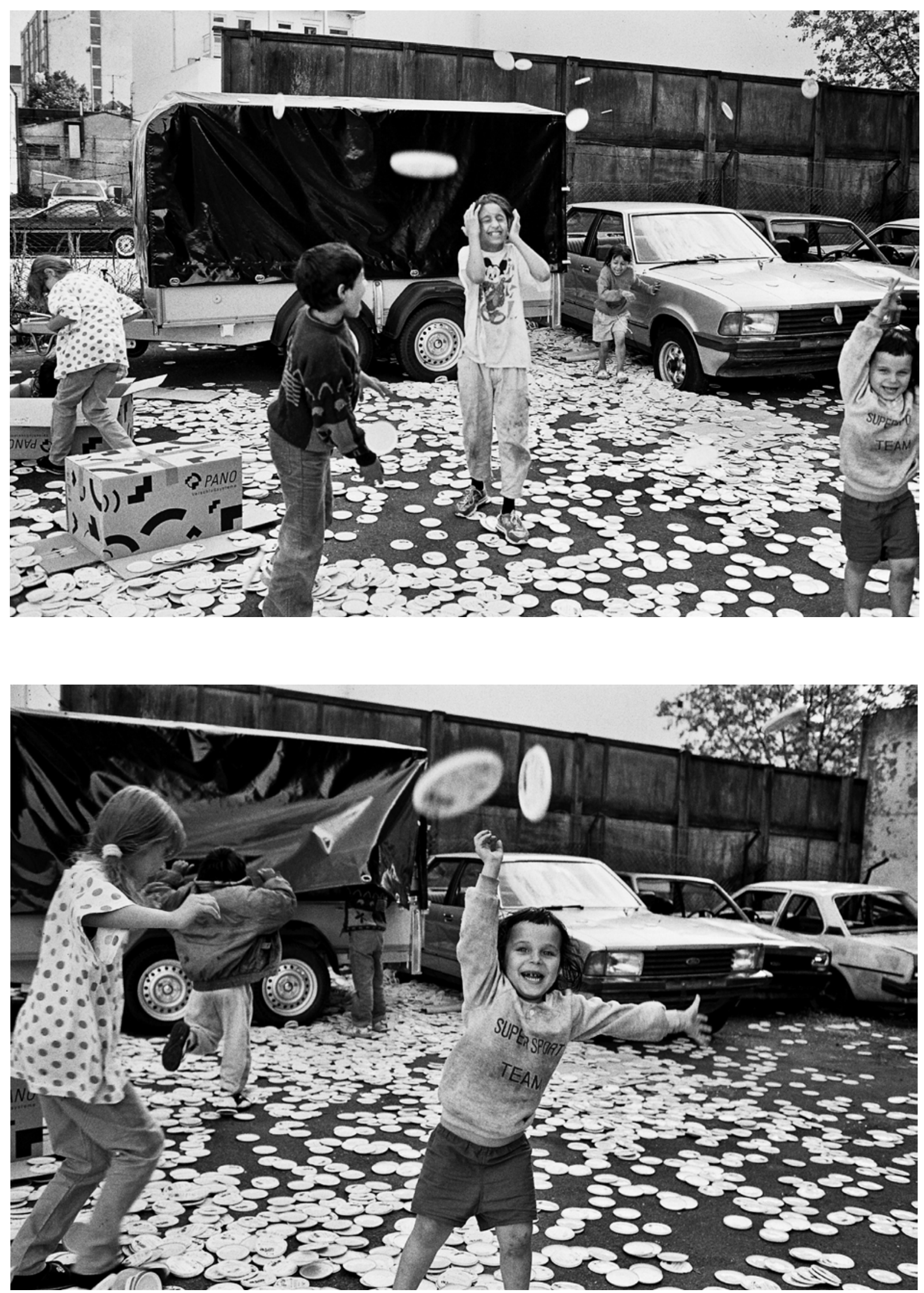
Manchmal habe ich mich auch länger mit den Kindern unterhalten, um genauer zu verstehen, was sie spielen - etwa mit dem koreanischen Jungen vor dem Laden seines Vaters in der Emilienstraße in Eimsbüttel, der Glassplitter von einer Autoscheibe aufsammelte. Ich fragte ihn, was er da mache und er sagte mir, dass er Diamanten sammle. Die Scherben glitzerten tatsächlich sehr schön. „Was willst du damit tun?“, fragte ich weiter. Er sagte, dass er sich mit den Diamanten etwas kaufen wolle; einen Computer wünsche er sich. Dann kam sein Vater und schrie ihn an, dass das keine Diamanten seien und er sofort aufhören solle, mit diesem „Dreck“ zu spielen.

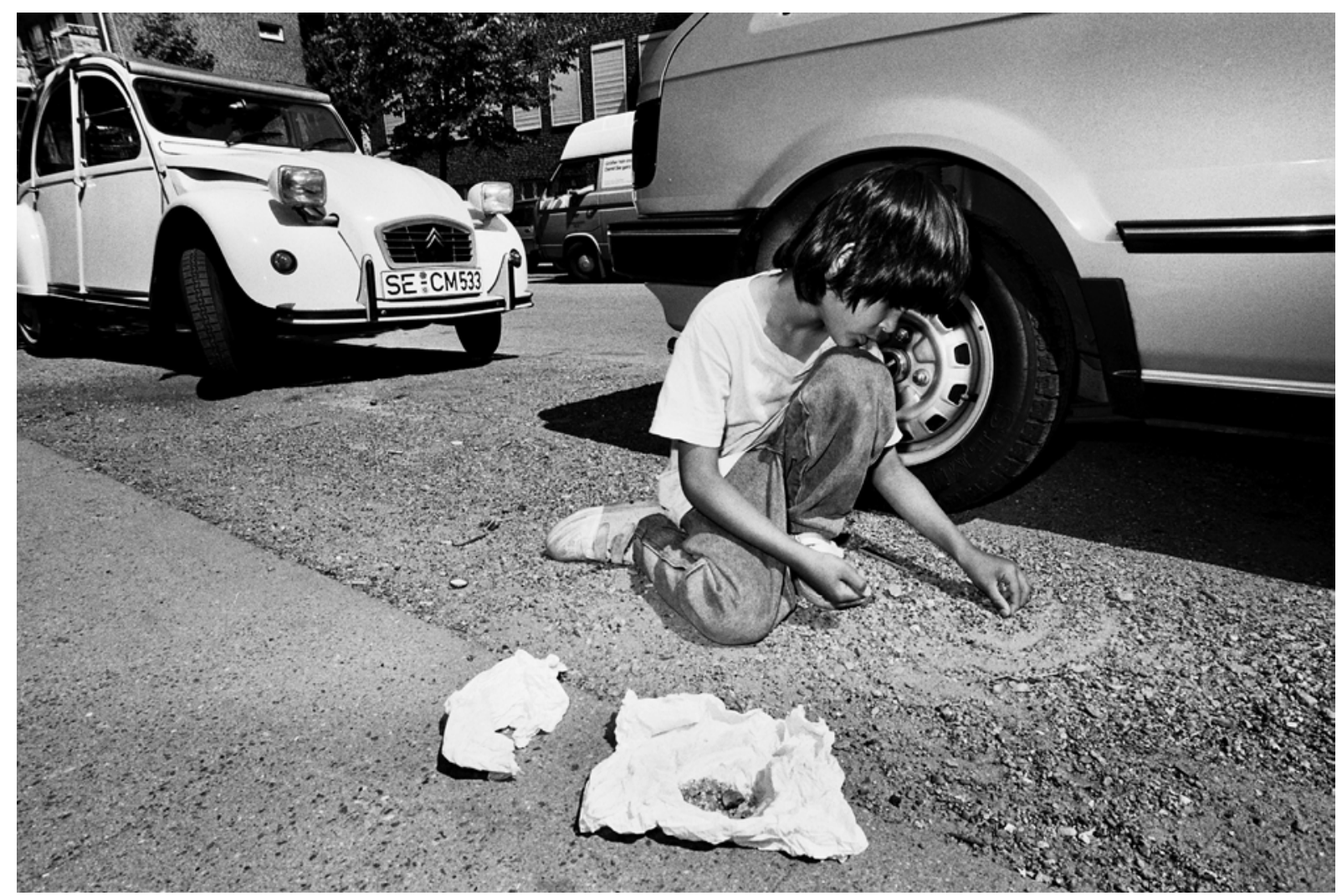


Oder die tägliche Rituale, die Kinder auf dem Nachhauseweg von der Schule vollzogen: Immer hielten sie an diesem Briefkasten an, um Telefon zu spielen.

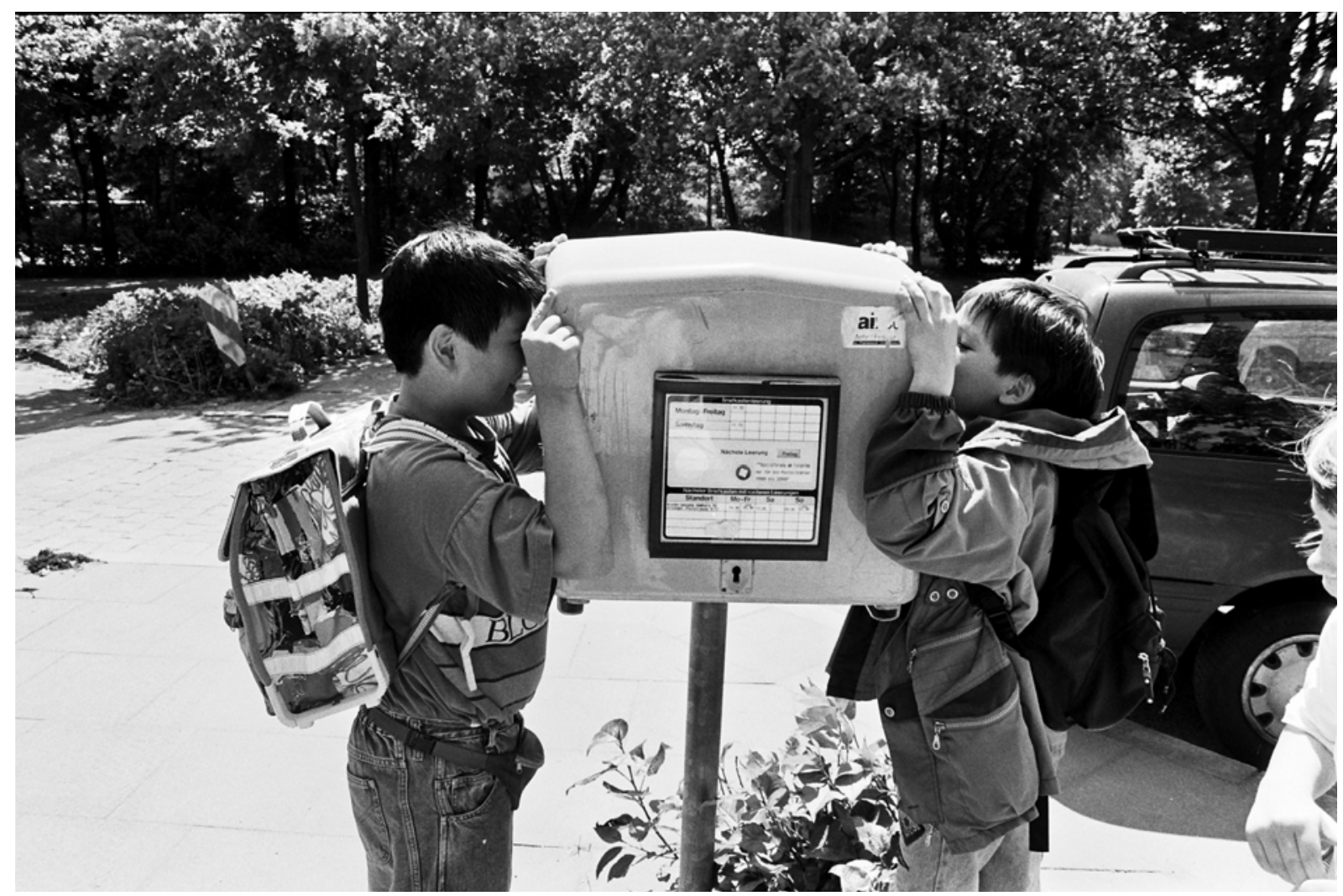

Bei der Roten Flora gab es früher eine Baugrube. Bei Regen wurde diese zu einem See. Dann benutzen die Kinder alte abgerissene Plakate als Unterlage und rutschten den Hügel hinunter.

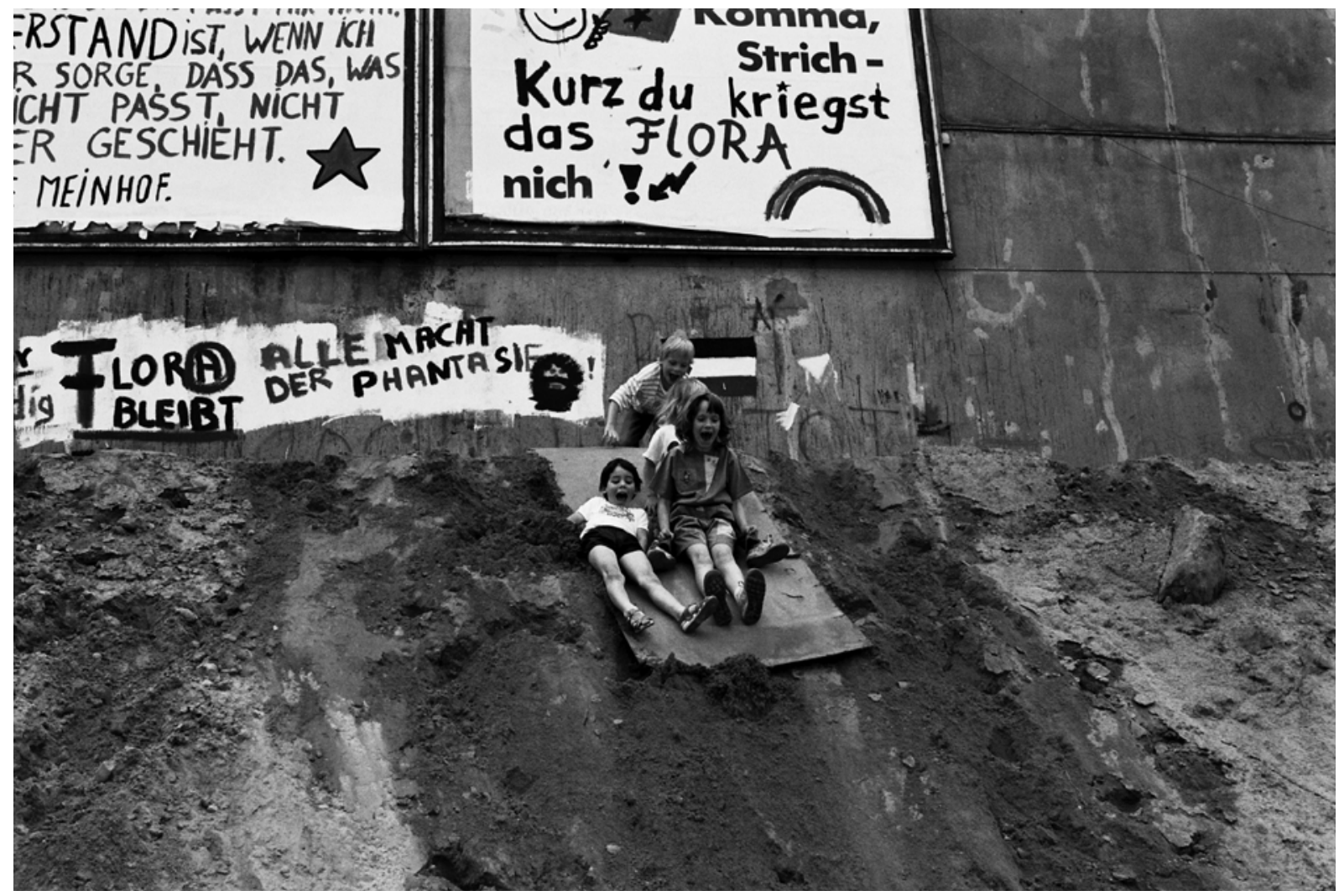



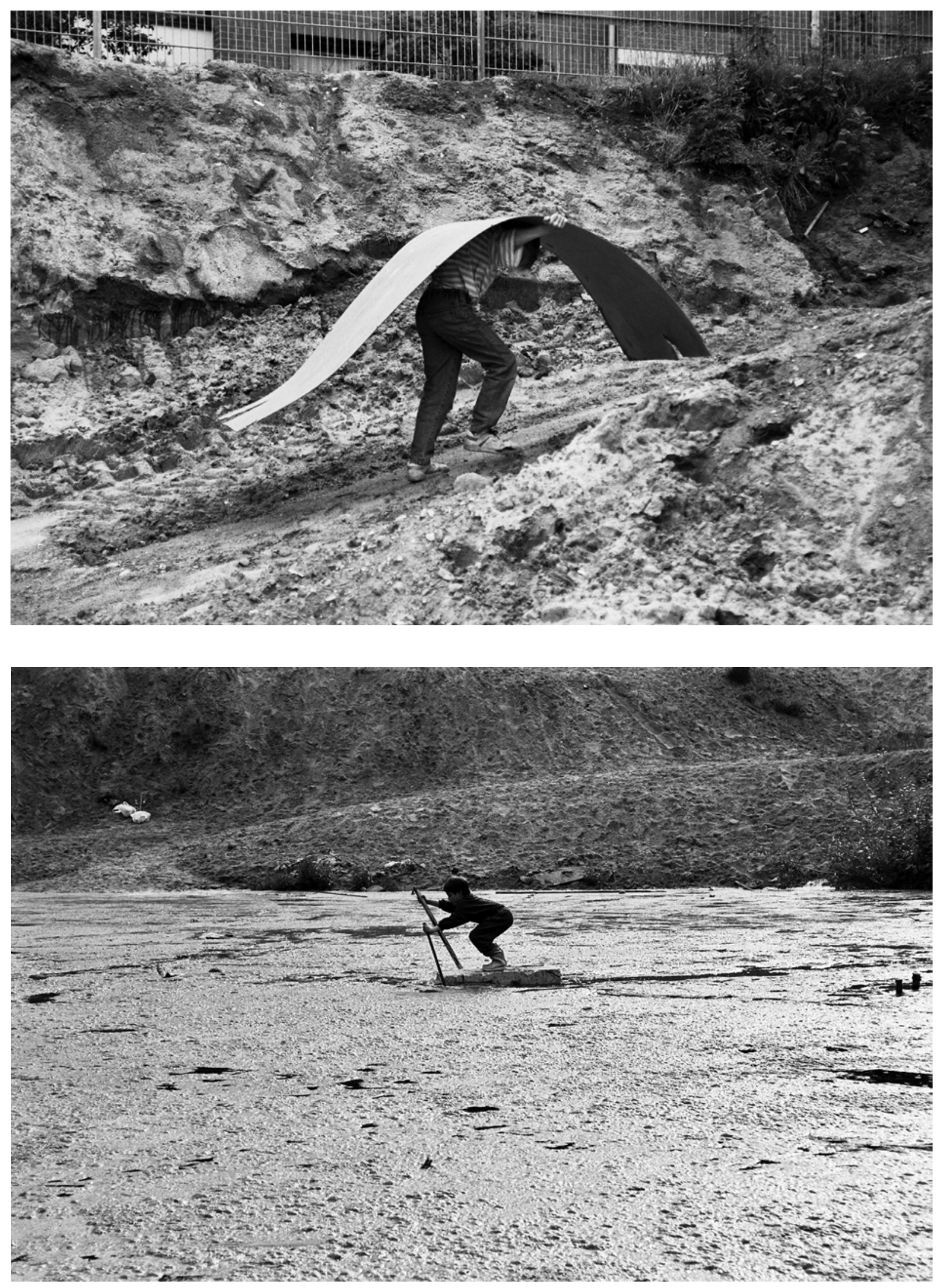
Kinder haben Fantasie und können aus Nichts wunderschönes Spielzeug erschaffen. Das liebe ich: umherlaufen und solche Dinge entdecken.

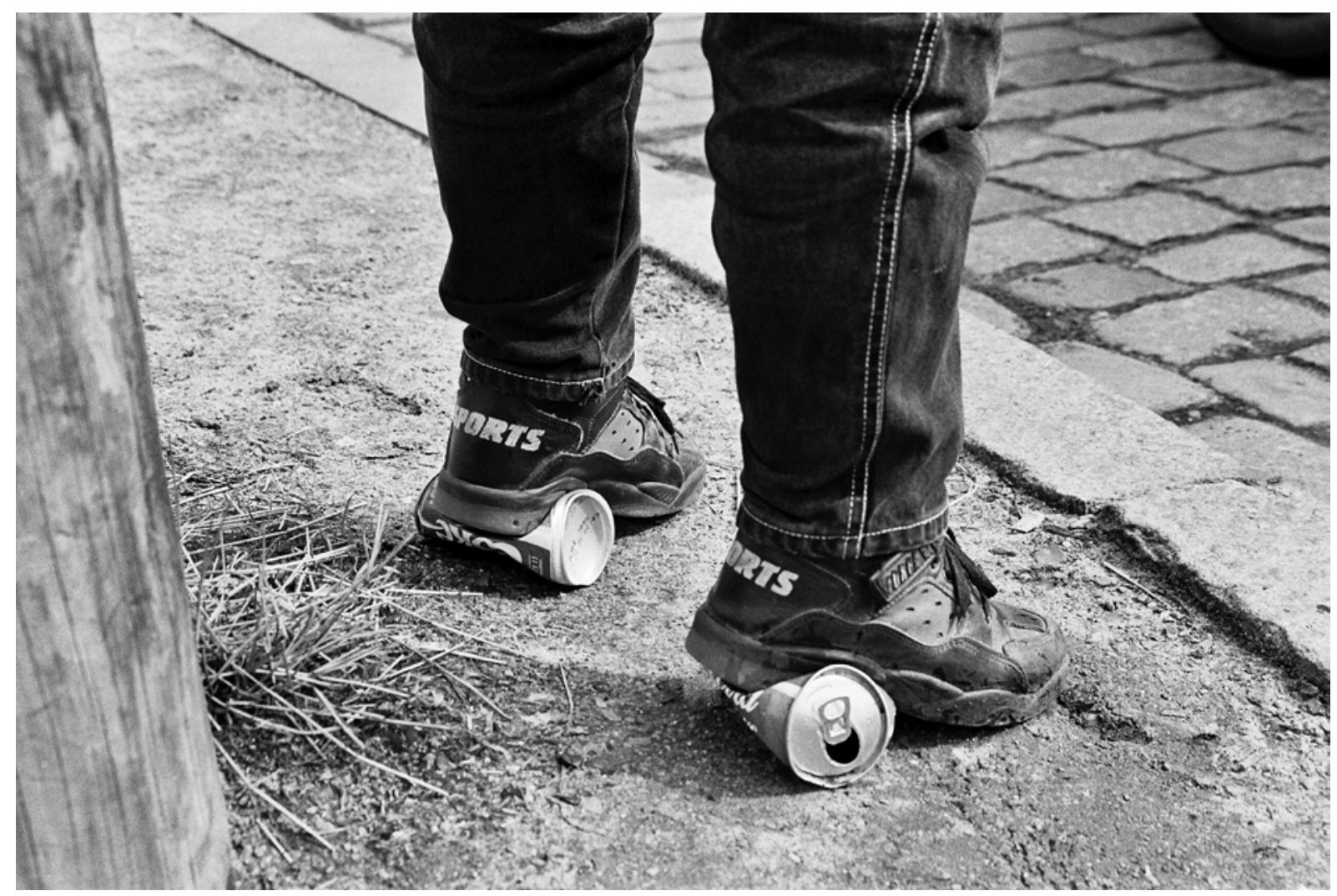

Irgendwann wurden diese verstreuten Aufnahmen zu einer Serie. Ich habe diese als Kalender veröffentlicht mit dem Titel Kinderspiele in der Stadt - Ihr habt die Uhren, wir haben die Zeit. Die Serie zu den Spielen in der Stadt ist also im Laufe der Jahre auf meinen Spaziergängen quasi nebenbei entstanden. Es war keine Auftragsarbeit!

Und das ist es auch, was mir an meiner Arbeit am besten gefällt: selber entscheiden zu können, was es wert ist, gezeigt zu werden und sich die Zeit nehmen zu dürfen, etwas so lange zu verfolgen, bis etwas Aussagekräftiges zusammenkommt. 
Heute sehe ich in Hamburg kaum noch Kinder auf der Straße spielen. Vielleicht findet das noch in kleinen Städten oder Dörfern statt. Aber in den griechischen Flüchtlingscamps, wo den Kindern kaum Spielzeug zur Verfügung steht, blüht noch die Fantasie. Für mich stellt sich daher die Frage, ob Kinder, die weniger Spielzeuge besitzen, mehr Spielraum für ihre Fantasie haben.
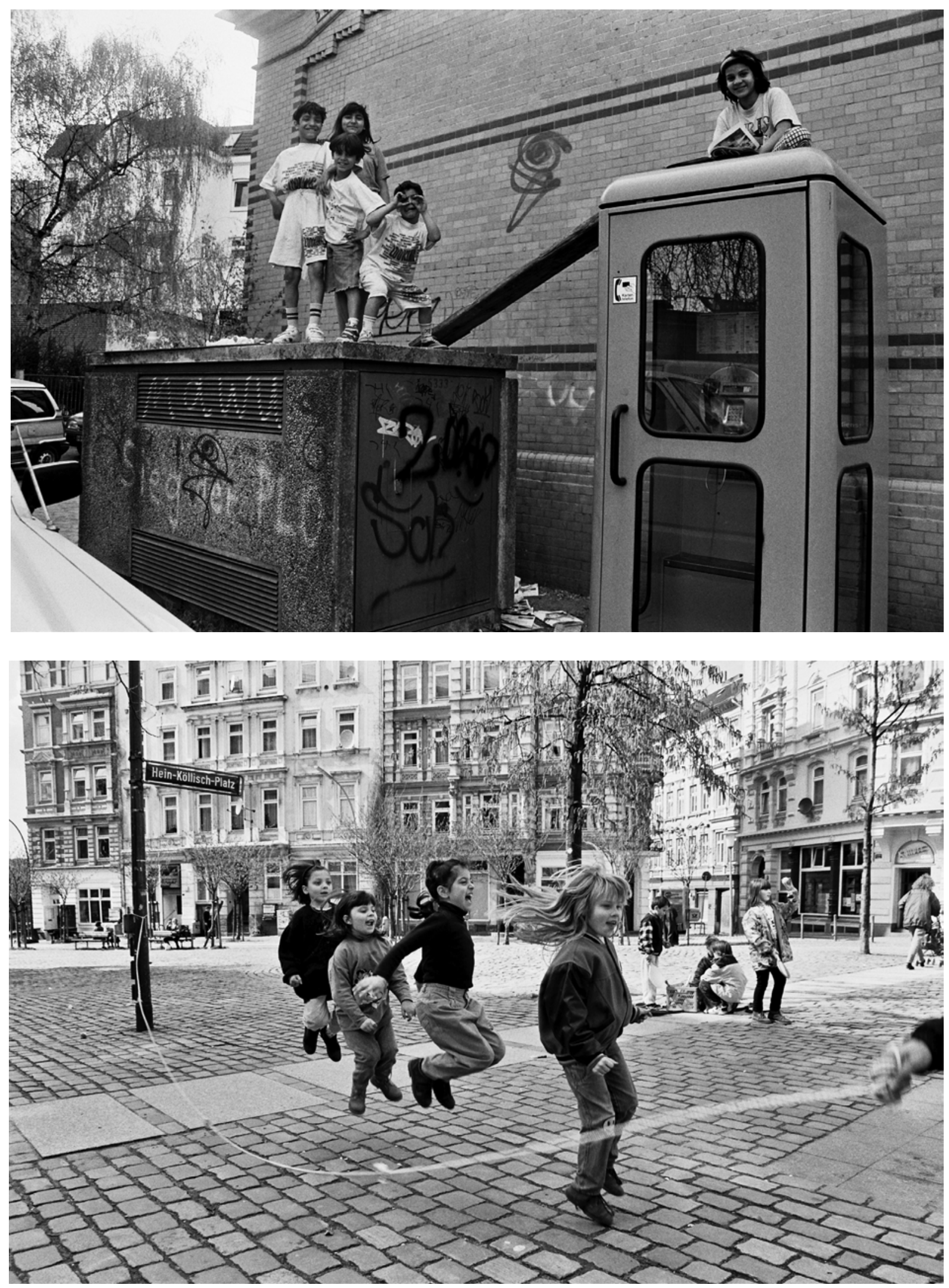


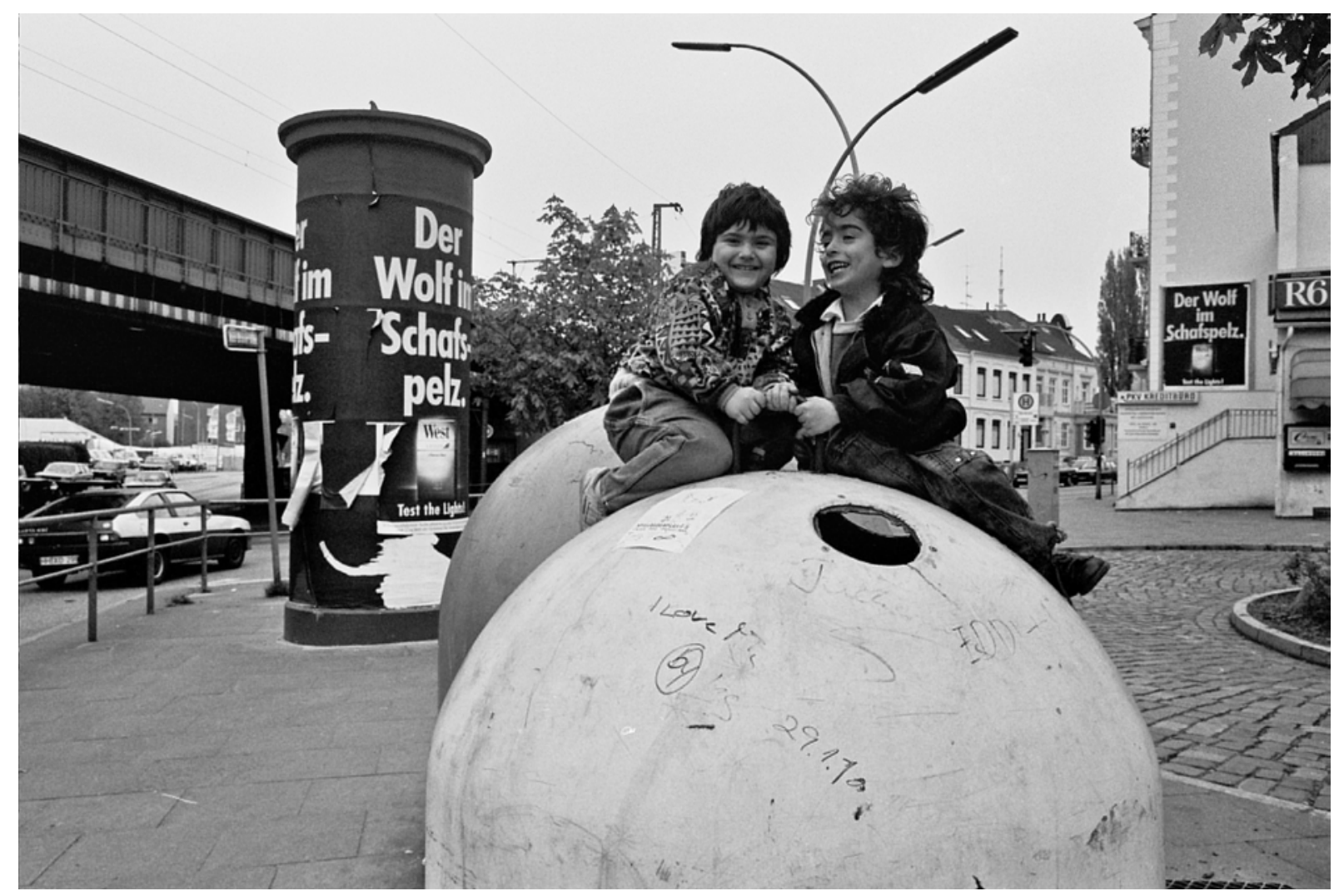

Welche Rolle spielt Dein politisches Engagement bei Deiner fotografischen Arbeit?

Meine fotografische Arbeit ist Spiegelbild meines politischen Engagements. Beides ist miteinander verschmolzen: Das eine geht nicht ohne das andere. Ich begreife mich als politischen Menschen und alles, was ich sehe, sehe ich durch diese Brille. Ich beobachte, was um mich herum geschieht, ich versuche andere zu verstehen und mische mich ein, um die Situationen, die ich beobachte, visuell einzufangen und festzuhalten. Außerdem nutze ich meine fotografischen Kenntnisse, um sie Jugendlichen und Kindern beizubringen; als ein Werkzeug, mit dem sie ihre Perspektiven auf die Welt selbstbestimmt kommunizieren können. „Solange die Löwen nicht ihre eigenen Historiker haben, werden die Jagdgeschichten weiter die Jäger verherrlichen“, lautet mein allerliebstes Sprichwort. Und das ist auch das Motto meiner Arbeit mit Kindern: Ich wünsche mir ganz viele Löwen. 


\section{Seit einigenJahren konzentriertsich Deine Arbeit vornehmlichauf Familien aufder Flucht oder Familien mit Migrationsgeschichte, wie Deine Projekte in Griechenland zeigen. Wie kam es dazu und welche Erfahrungen hast Du in diesen Arbeiten gemacht?}

Ich arbeite seit über 30 Jahren mit Flüchtlingskindern und Fotografie (vgl. mein Buch Bei mir ist alles normal, das 2004 im Brandes \& Apsel-Verlag erschienen ist). Mit den Flüchtlingsschiffen im Hamburger Hafen fing alles an: Hier mussten neu ankommende Familien anfangs leben. Es gab eigentlich nichts für Kinder. Gemeinsam mit weiteren antirassistischen Gruppen begannen wir damals, nachmittags in einem Zelt vor den Schiffen mit den Kindern zu spielen.

Später entwickelte sich daraus ein richtiges Programm, das eine Terre des Hommes-Gruppe für die Kinder auf den Schiffen anbot. In diesem Rahmen fing ich an, gemeinsam mit dem Psychologen Reimer Dohrn Fotoworkshops mit Kindern zu machen, speziell mit Kindern, die „freiwillig“ zurückkehren mussten, zum Beispiel nach Bosnien oder in den Kosovo oder mit Kindern, die aus Kriegsregionen kamen. Manche der damals sehr jungen Angekommenen sind heute noch meine Freund*innen. Sie sind mittlerweile zum Teil verheiratet, haben eigene Kinder oder arbeiten mit im Wohnschiffprojekt e. V.

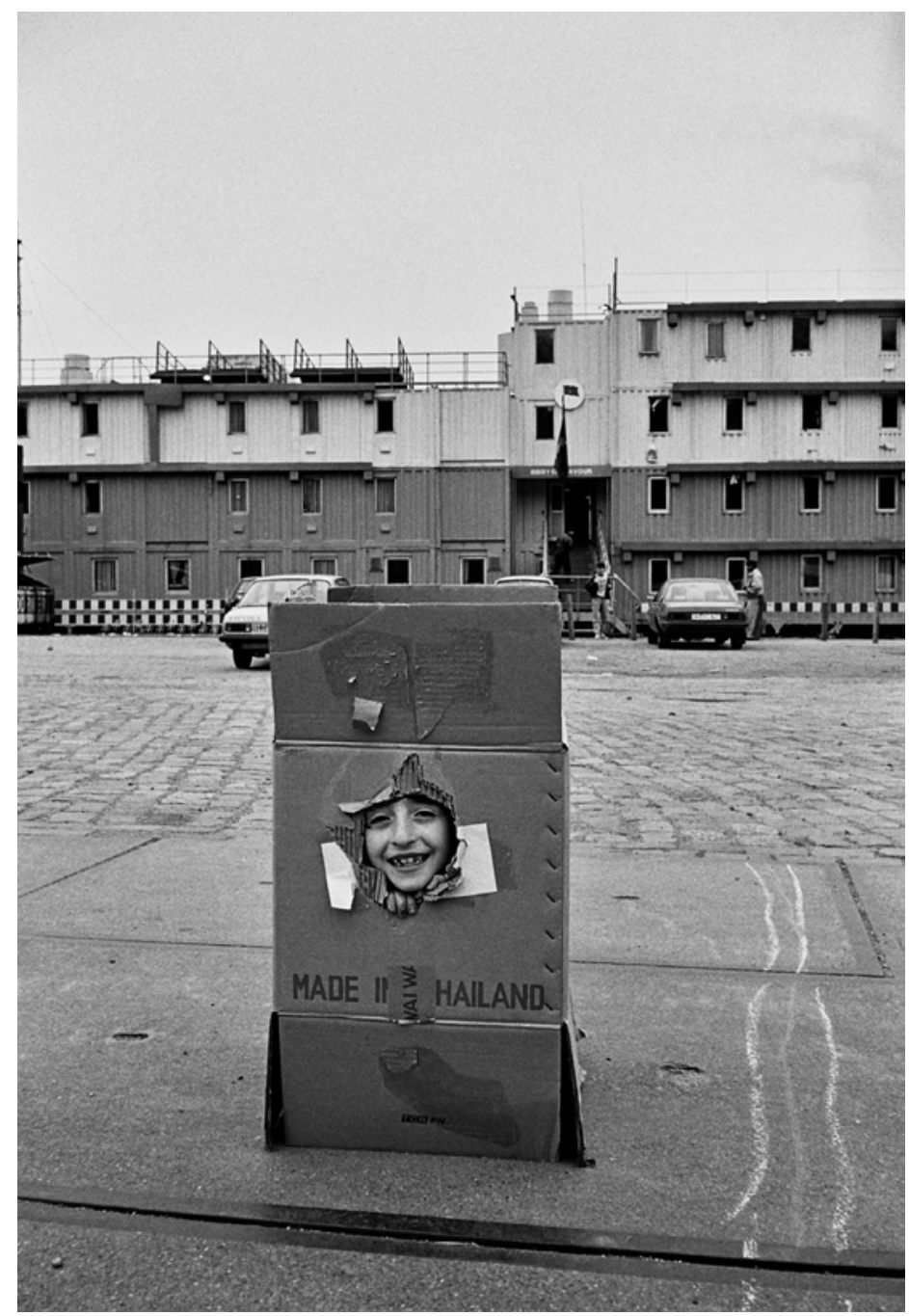


Nach über zehn Jahren Fotoworkshops habe ich angefangen, diese Lebensgeschichten zu dokumentieren, beispielsweise mit dem kleinen Buch Ich will mein baba back. Hier erzählt eine Mutter mit ihren vier Kindern ihre Fluchtgeschichte. Während ich zuhörte, malten die Kinder ihre Erinnerungen. Daraus haben wir ein Büchlein gemacht, das für die Öffentlichkeit gedacht war und ihren Vater aus dem Gefängnis herausbekommen sollte - was auch gelungen ist.

Ein weiteres Buch entstand mit Arjan: Als damals 9-Jährige saß sie in den Schulferien oft bei mir und langweilte sich. Dann fing sie an, die Geschichte ihrer Flucht aufzuschreiben. Erst mal waren es zwei Seiten, dann habe ich ihr vorgeschlagen, mit ihrer Familie darüber zu sprechen, um auch deren Erinnerungen aufzunehmen. Am Schluss wurden es 50 Seiten. Arjan ergänzte noch Zeichnungen und schließlich haben wir die Geschichte als kleines Buch drucken lassen. Die Einnahmen konnte Arjan behalten. Sie ist mittlerweile verheiratet, selbst Mutter und berufstätig. Auf Rundfahrten im Hamburger Hafen liest sie zusammen mit Reimer Dohrn seit Jahren aus ihrem Buch vor.

Oder Parwana: Sie musste viele Jahre im „Hotspot“ Moria leben und bat mich, mit ihr ein Buch darüber zu machen. 2019 ist Theolivetreeand theold woman erschienen - ein kleines Buch, das auf Englisch, Deutsch, Griechisch und Französisch übersetzt wurde (Amiri 2019). Ich habe über den Prozess der gemeinsamen Arbeit einen Film mit Zeichnungen, Fotos und Home Videos gemacht, der gerade fertig geworden ist (Stroux 2021).

Abschließend kann ich sagen, dass es mich glücklich macht, Jugendliche und Kinder dadurch zu stärken, dass ich ihnen die Möglichkeiten der Fotografie aufzeige und ihnen Mut mache, ihre eigenen Geschichten zu schreiben.

Das Gespräch führten Stefan Höhne und Verena Schreiber.

\section{Autor_innen}

Marily Stroux ist Fotografin und lebt in Hamburg. Seit den 1980er Jahren begleitet sie mit ihrer Kamera dort das Alltagsleben und die zahlreichen stadtpolitischen Kämpfe. In den letzten Jahren arbeitet sie vor allem mit Familien auf der Flucht, beispielsweise im Flüchtlingslager Moria auf der griechischen Insel Lesbos.

\section{Literatur}

Amiri, Parwana (2019): The olive tree and the old woman. A conversation in the olive grove of Moria hotspot. http://lesvos.w2eu.net/files/2020/o3/Pixi-Baum-II_FA_DRUCK.pdf (letzter Zugriff am 4.10.2021).

Stroux, Marily (2021): The olive tree. 31.5.2021. https://www.youtube.com/watch?v=UG_ qX63-e3Q (letzter Zugriff am 4.10.2021). 
